\title{
SERUM LACTATE AS A NOVEL POTENTIAL BIOMARKER IN MULTIPLE SCLEROSIS
}

Angela M. Amorini, ${ }^{\mathrm{a}}$ Viviana Nociti, ${ }^{\mathrm{b}}$ Axel Petzold, ${ }^{\mathrm{c}}$ Claudio Gasperini, ${ }^{\mathrm{d}}$ Esmeralda Quartuccio, ${ }^{\mathrm{d}}$ Giacomo Lazzarino, ${ }^{\mathrm{a}}$ Valentina Di Pietro, ${ }^{\mathrm{e}}$ Antonio Belli, ${ }_{\mathrm{e}}^{\mathrm{e}}$ Stefano Signoretti, ${ }^{\mathrm{d}}$ Roberto Vagnozzi ${ }^{\mathrm{f}}$ Giuseppe Lazzarino, ${ }^{\mathrm{g} *}$ Barbara Tavazzi ${ }^{\mathrm{a}}$

anstitute of Biochemistry and Clinical Biochemistry, Catholic University of Rome, Italy

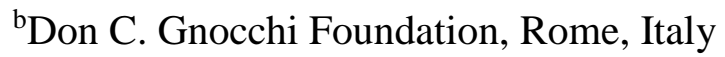

${ }^{\mathrm{c}}$ Department of Neurology, VU Medical Centre, Amsterdam, The Netherlands

${ }^{\mathrm{d} D e p a r t m e n t ~ o f ~ N e u r o s c i e n c e s, ~ S ~ C a m i l l o ~ F o r l a n i n i ~ H o s p i t a l, ~ R o m e, ~ I t a l y ~}$

${ }^{\mathrm{e}}$ Neuropharmacology and Neurobiology Section, School of Clinical and Experimental Medicine,

College of Medical and Dental Sciences, University of Birmingham, Birmingham, UK.

fDepartment of Biomedicine and Prevention, Section of Neurosurgery, University of Rome "Tor Vergata", Rome, Italy.

gDepartment of Biology, Geology and Environmental Sciences, Division of Biochemistry and Molecular Biology, University of Catania, Catania, Italy.

*Correspondence should be addressed to: Prof. Giuseppe Lazzarino

Department of Biology, Geology and Environmental Sciences

Division of Biochemistry and Molecular Biology

University of Catania, Viale A. Doria 6, 95125 Catania, Italy

Tel.: +39-0957384095; Fax: +39-095337036; E-mail: lazzarig@unict.it

Running title: Serum lactate in MS

Text word count $=4,019$ 


\section{ABSTRACT}

Multiple sclerosis (MS) is a primary inflammatory progressive demyelinating disorderdisease associated with a probably secondary progressive neuronegenerative component. , probably caused by an autoimmune attack towards myelin. MS is characterized by mImpaired mitochondrial malfunctioning has been hypothesised to drive neurodegeneration and called, the "virtual hypoxia hypothesis" imbalancing adenosine triphosphate production and consumption and leading to cell energy deficit. Biochemical data of support to this hypothesis comes from Previous data showed that MS patients have increased lactate levels in both the brain tissue and cerebrospinal fluid studies with elevated lactate levels which suggest an increased anaerobic metabolism in MS. The aAim of thisour multicentre study was to determinetest whetherif this data could be extended to blood lactate measurements in MS patients who fulfilled the 2005 revision of the MacDonald criteria for MS as well as healthy control subjects.had values of circulating lactate different from those of controls. We included serum samples from 1851 pPatients (1226[1] MS, 625 controlsn = 613) from three centers, were recruited at the Catholic University of Rome, Italy, $(n=281)$, at the MS Centre Amsterdam, The Netherlands $(\mathrm{n}=158)$ and at the S Camillo Forlanini Hospital, Rome, Italy $(\mathrm{n}=$ 174). Clinical disability was assess They were clinically assessed using the Extended Disability Status Scale score (EDSS) and classified clinically according to Lublin and Reingold. Peripheral blood was used to determine sSerum lactate levels were quantified spectrophotometrically with the anlyst being blinded to all clinical information., using the lactate oxidase-based spectrophotometric method. ISerum lactate in patients with MS patients serum lactate levels was about were three times higher $(3.04 \pm 1.26 \mathrm{mmol} / \mathrm{l})$ than that of compared to serum lactate levels of 625 matched healthy controls $(1.09 \pm 0.25 \mathrm{mmol} / \mathrm{l}, \mathrm{p}<0.0001)$. Importantly, serum lactate levels were increase across When divided according to their clinical groups with higher levels in cases with a progressive disease course compared to those with a characteristics, MS patients had serum lactate values 
related to the clinical course ( relapsing-remitting, disease course secondary progressive and primary progressive). In addition, there was a serum lactate and the EDSS scores were linearly correlation between serum lactate levels and the EDSS ed $\left(\mathrm{R}^{2}=0.419 ; \mathrm{p}<0.001\right)$. These data strongly suggest that $\mathrm{M}$ support the hypothesis that mitochondrial dysfunction is an important feature in MS and of particular relevance to the neurodegenerative phase of the disease. is characterized by energy penalty and indicate that the determination of circulating Measurement o0f serum lactate in MS might be a relative inexpensive test for relevant for longitudinal monitoring of "virtual hypoxia" in MS. Serum lactate may also be a secondary outcome for the disease progression and the efficacy of treatments trials aimed to improve mitochondrial function in patients with MS.drug treatments, using an inexpensive, easy to perform, low invasive analytical parameter.

Keywords: clinical disability; energy penalty; mitochondrial dysfunction; multiple sclerosis; serum lactate.

Abbreviations: $\mathrm{CPK}-\mathrm{BB}=$ creatinphosphokinase brain specific isoform; $\mathrm{CrP}=$ creatine phosphate; EDSS $=$ expanded disability scale $;$ ETC $=$ electron transport chain; ${ }^{1} \mathrm{H}-\mathrm{MRS}=$ proton-magnetic resonance spectroscopy; $\mathrm{MS}=$ multiple sclerosis; $\mathrm{PP}=$ primary progressive; $\mathrm{RNS}=$ reactive nitrogen species; $\mathrm{ROS}=$ reactive oxygen species; $\mathrm{RR}=$ relapsing remitting; $\mathrm{SP}=$ secondary progressive. 


\section{INTRODUCTION}

Multiple sclerosis (MS) is a progressive demyelinating disorder central nervous system causing severeaccumulation of disability to the patients up to their death. MS is believed to be an autoimmune pathology (Pineas and Parratt, 2012) although the reasons for the autoimmune attack towards myelin are not yet totally clear (Brassar, 2012). As it occurs in other chronic neurodegenerative disorders (Alzheimer's disease, Parkinson's disease), MS is characterized by various molecular alterations including change in ionic homeostasis (Ehling et al., 2011; Stadelmann, 2011), overproduction of reactive oxygen (ROS) and nitrogen species (RNS), with consequent oxidative/nitrosative stress and induction of apoptosis (Seven et al., 2013; Fiorini et al., 2013; Haider et al., 2011). All these events seriously compromise many fundamental neuronal functions and certainly play a role in the disease progression and worsening of the patients' clinical conditions. In addition, these molecular changes seem to have, as a common feature, the malfunctioning of mitochondria leading to formulate the "mitochondrial hypothesis" to explain axonal degeneration in MS (Su et al., 2009; Su et al., 2013; Cambron et al., 2012), as well as to formulate the concept of "virtual hypoxia" in which chronically demyelinated axons of MS patients are forced to operate (Trapp and Stys, 2009).

This hypothesis is supported by many experimental and clinical data showing that MS is characterized by a remarkable energy penalty due to the impairment between energy production and consumption (Trapp and Stys, 2009; Cambron et al., 2012; Hattingen et al., 2011). This imbalance is determined by a decreased mitochondrial ability to supply adequate ATP concentrations for the various energy-dependent functions crucial for the neuronal survival. In particular, imbalance in creatinphosphate (CrP) homeostasis (Tur et al., 2012) and decreased activity of the brain specific isoform of the enzyme creatinphosphokinase (CPK-BB) detected in MS (Steen et al., 2010), should be responsible for the reduced export of the mitochondrially generated ATP to the cytoplasm, with 
consequent decreased availability of cytoplasmic ATP. Contribution to a net decrease in ATP cellular content should also be due to an impairment in the activity of the mitochondrial electron transport chain (ETC). In fact, it has been demonstrated that MS patients have decreased expression of various subunits of complexes I, III, IV and V of the ETC in different brain regions (Pandit et al. 2009), with consequent diminution in the electron flow through the chain and inevitable decrease in ATP formation by the electron-dependent oxidative phosphorylation. The overall decline in cytoplasmic ATP negatively influences all the ATP-dependent reactions, including the activity of ATP-ases involved in ionic homeostasis (Dutta et al., 2006), causing imbalance in intracellular calcium and membrane depolarization (Dutta et al., 2006; Andrews et al., 2005).

In line with the hypothesis of mitochondrial malfunctioning, we have previously demonstrated that MS patients have increased levels of compounds deriving from ATP catabolism (hypoxanthine, xanthine, uric acid, uridine, creatinine) in their cerebrospinal fluid (CSF) and blood (Amorini et al., 2009; Lazzarino et al., 2010; Tavazzi et al., 2011). These data strongly supported the concept that MS patients have impaired energy metabolism, with significant imbalance in the production and consumption of ATP (Steen et al., 2010; Tur et al., 2012) ultimately causing an overproduction of its catabolites in the cerebral tissue (Langemann et al., 1992). Thanks to their ability to freely cross the neuronal membrane, these low molecular weight compounds are initially released into the CSF of MS patients, subsequently reaching the blood stream, and finally leading to a significant raise in their concentrations in the biological fluids respect to values found in healthy controls (Amorini et al., 2009; Lazzarino et al., 2010; Tavazzi et al., 2011). With this current knowledge of an energy penalty in MS, it is reasonable to imagine that, in order to counteract the diminished ATP supply caused by altered mitochondrial functions, cerebral cells may tend to increase the glycolytic pathway to rates exceeding the already compromised capacity of mitochondria to metabolize pyruvate. In consequence of an increased glycolytic rate and pyruvate accumulation an increase in lactate production might be expected. Recently, by using proton-magnetic resonance spectroscopy 
$\left({ }^{1} \mathrm{H}-\mathrm{MRS}\right)$, it has been shown that MS patients had increased lactate in their CSF, suggesting increased extra-mitochondrial glucose metabolism due to mitochondrial dysfunction (Regenold et al., 2008). A correlation between lactate concentration in CSF and the number of inflammatory plaques reinforced the indication of increased glycolysis in MS due to mitochondrial malfunctioning (Simone et al., 1996; Lutz et al., 2007). Notwithstanding, data reporting decrease in CSF lactate in the early stages of MS have also been published (Fonalledas Perelló et al., 2008), thereby rendering unclear the importance of measuring this compound. Furthermore, to increase the uncertainty, additional ${ }^{1} \mathrm{H}-\mathrm{MRS}$ studies indicated either elevated (Zaaraoui et al., 2010) or no change (Schocke et al., 2003) of brain lactate in MS patients. To date, no data are available concerning the concentration of circulating lactate in MS patients. Interestingly, a retrospective evaluation of the chromatographic runs of serum samples of MS patients enrolled in our previous studies (Amorini et al., 2009; Tavazzi et al., 2011) showed an impure peak having the retention time of true lactate which, in the majority of samples, was much higher than that found in controls (data not shown).

In this study, we measured the concentration of serum lactate in a large cross-sectional cohort of MS patients, recruited in three different Centers, comparing the results with those recorded in a group of control, age and sex matched, healthy subjects. The hypotheses tested were firstly that an energy deficit in MS would lead to higher lactate levels, secondly that any rise of serum lactate in MS would be clinically relevant and related to clinical disability and disease course. 


\section{MATERIALS AND METHODS}

\section{Selection and clinical evaluation of the MS patients}

Patients ( $\mathrm{n}=613$ ) fulfilling the 2005 revision of the diagnostic panel criteria for MS (Polman et al., 2005) were recruited at the Institute of Neurology of the "Policlinico Gemelli" of the Catholic University of Rome (center $1 ; \mathrm{n}=281$ ), at the Department of Neurosciences, S Camillo Forlanini Hospital, Rome, Italy (center 2; $n=174$ ) and at the Department of Neurology, VU Medical Centre, Amsterdam, The Netherlands (center 3; $\mathrm{n}=158$ ). They were clinically assessed using the Extended Disability Status Scale score (EDSS) (Krutzke, 1983). In coincidence with the medical examination, patients were asked to undergo to a blood withdrawal to be used for this research study. Only patients that at the moment of the blood collection were clinically stable were included in the study. A clinical relapse within one month before withdrawal was used as an exclusion criterion.

Patients were classified into relapsing remitting (RR), secondary progressive (SP) or primary progressive (PP), according to Lublin and Reingold (Lublin and Reingold, 1996). The control group consisted of 625 healthy subjects, matched for age and gender, and recruited among the students of the Catholic University of Rome and the University of Catania, as well as among the personnel of these two Universities who underwent the annual health check-up. Subjects suffering from any acute or chronic systemic disease, which might have influenced the serum lactate levels, were not included in the control group. The study was approved by the local Ethic Committees and written informed consent was obtained from all patients according to the Declaration of Helsinki.

\section{Preparation of samples}

Peripheral venous blood samples were collected, using the standard tourniquet procedure, from the antecubital vein into a single VACUETTE $^{\circledR}$ polypropylene tube containing serum separator and clot activator (Greiner-Bio One GmbH, Kremsmunster, Austria). Blood withdrawals in both controls 
and MS patients were carried out after at least 15 minutes of complete rest. After 30 minutes at room temperature, samples were centrifuged at $1890 \mathrm{x} g$ for $10 \mathrm{~min}$ to separate sera. To measure lactate concentration, an aliquot of all serum samples was used with no further processing. This protocol for blood withdrawal and serum preparation was strictly observed in the three centers involved and was equally used either in all controls or in all MS patients.

\section{Spectrophotometric assay of serum lactate}

The spectrophotometric determination of lactate was carried out using an Agilent 89090A spectrophotometer (Agilent Technologies, Santa Clara Ca, USA) and following the method described by Artiss et al. (Artiss et al., 2000). Briefly, the reaction mixture contained $100 \mathrm{mM}$ Tris$\mathrm{HCl}, 1.5 \mathrm{mM}$ N-ethyl-N-2-hydroxy-3-sulfopropyl-3-methylalanine, $1.7 \mathrm{mM}$ 4-aminoantipirine, 5 IU horseradish peroxidase. Fifty $\mu \mathrm{l}$ of serum were added to the mixture, let to stand for 5 min and read at $545 \mathrm{~nm}$ wavelength. The reaction was started with the addition of $5 \mathrm{IU}$ of lactate oxidase to the cuvette (finale volume $=1 \mathrm{ml}$ ) and it was considered ended when no change in absorbance were recorded for at least $3 \mathrm{~min}$. To calculate lactate in serum samples, the difference in absorbance at $545 \mathrm{~nm}$ wavelength $\left(\Delta_{\text {abs }}\right)$ of each sample was interpolated with a calibration curve obtained by plotting $\Delta_{\text {abs }}$ measured in standard solutions of lactate with increasing known concentrations To compare analytical results of serum lactate obtained with the afore described method, 150 samples from the control group and 150 samples from the groups of MS patients were randomly selected and assayed at the "Policlinico Gemelli" Hospital of the Catholic University of Rome, applying the same lactate oxidase-based method and using conventional apparatus routinely used in the clinical biochemistry laboratory of the hospital (Cobas c 702, Roche Diagnostics). 


\section{Statistical analysis}

Normal data distribution of data in controls and MS patient was assessedtested using by the Kolmogorov-Smirnov test. For normally districuted data, Dgroup differences between the two groups were assessed by the Student's t-test for unpaired observations. Due to the very different number of subjectsunbalanced study design the Kruskal-Wallis one-way ANOVA by ranks, followed by the Friedman test was used for comparison of , significances among controls and MS subgroups of MS on clinical MS subtypes (RR, SP, PP) were assessed by the and controls.KruskalWallis one-way ANOVA by ranks, followed by the Friedman test. CAnalysis of correlation and regression analyses fo serum lactate levels with the EDSS were, followed by ANOVA of the regression coefficients, was performed to estimate and test serum lactate of MS patients as a function of the EDSS scores. Only A two-tailed p-values of $\mathrm{p}<$ less than 0.05 wereas considered as statistically significant. 


\section{RESULTS}

The demographic and clinical data of the group of control healthy subjects and of MS patients enrolled in this study are summarized in Table 1. When subgrouped on the basis of the EDSS scores 64 patients were EDSS 0, 72 EDSS 1, 26 EDSS 1.5, 76 EDSS 2, 25 EDSS 2.5, 75 EDSS 3, 37 EDSS 3.5, 58 EDSS 4, 16 EDSS 4.5, 26 EDSS 5, 10 EDSS 5.5, 72 EDSS 6, 20 EDSS 6.5, 24 EDSS 7, 6 EDSS 7.5 and 6 EDSS 8. The majority of patients with MS had a RR disease course ( $\mathrm{n}=430$; $70.1 \%)$, with smaller numbers of them being affected by the SP $(n=153 ; 25.0 \%)$ and the PP $(n=$ $30 ; 4.9 \%$ ) forms. The group of RR-MS patients had significantly lower mean values of EDSS and disease duration compared to the corresponding values recorded in the groups of both SP-MS and PP-MS patients $(\mathrm{p}<0.01)$.

Table 1. Clinical neurolog[2]ical data of MS patients and controls. N/A = not available

\begin{tabular}{cccccc}
\hline & $\begin{array}{c}\text { CTRL } \\
(\mathbf{n}=\mathbf{6 2 5})\end{array}$ & $\begin{array}{c}\text { MS (all) } \\
(\mathbf{n}=\mathbf{6 1 3})\end{array}$ & $\begin{array}{c}\text { RR } \\
(\mathbf{n}=\mathbf{4 3 0})\end{array}$ & $\begin{array}{c}\text { SP } \\
(\mathbf{n}=\mathbf{1 5 3})\end{array}$ & $\begin{array}{c}\text { PP } \\
(\mathbf{n}=\mathbf{3 0})\end{array}$ \\
\hline Age (years) & $44.8 \pm 11.7$ & $45.4 \pm 12.8$ & $43.1 \pm 12.5$ & $49.7 \pm 11.7$ & $51.3 \pm 13.4$ \\
& $46.0(15-73)$ & $46.5(15-80)$ & $42.0(15-70)$ & $50.2(28-72)$ & $53.0(26-80)$ \\
\hline Gender & F $420:$ M 205 & F $411:$ M 202 & F $276:$ M 154 & F $110:$ M 43 & F $21:$ M 9 \\
\hline Disease duration & N/. A. & $12.9 \pm 9.5$ & $10.8 \pm 9.7$ & $18.8 \pm 10.0$ & $14.8 \pm 9.8$ \\
\hline Edears $)$ & & $12.0(0-44)$ & $9.0(0-38)$ & $18.2(1-44)$ & $15.1(0.6-35)$ \\
\hline & N/. A. & $3.3 \pm 2.1$ & $2.1 \pm 1.6$ & $5.8 \pm 1.5$ & $5.2 \pm 1.5$ \\
& & $3.0(0-8)$ & $2.0(0-8)$ & $6.0(0-8)$ & $6.0(3.0-8)$ \\
\hline
\end{tabular}

Values are expressed as mean \pm S.D. and median (range). CTRL $=$ controls; MS (all) $=$ total number of multiple sclerosis patients; PP = Primary Progressive; SP = Secondary Progressive; RR = Relapsing Remitting; N.A. = not assessed; EDSS = Extended Disability Status Scale score. 
Data referring to the concentration of circulating lactate detected in serum of controls and MS patients are illustrated in Figure 1[3].
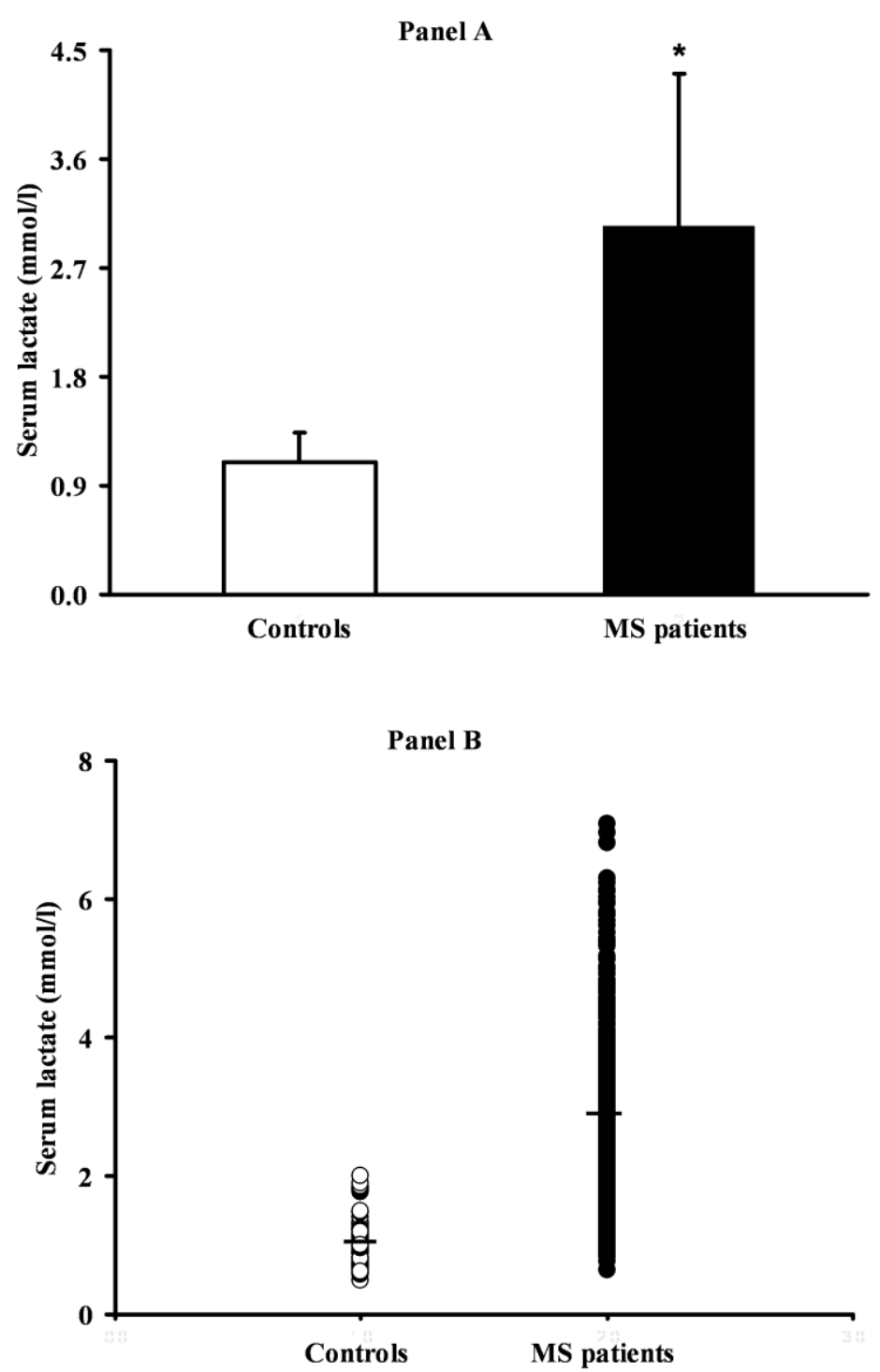

Figure 1. Concentration of serum lactate determined in peripheral venous blood samples of 625 control healthy subjects and 613 MS patients. In Panel A, mean values with standard deviations represented by vertical bars, are reported. In Panel B, the individual values of each subject of both groups, with medians represented by horizontal marks (1.10 and 2.87 in controls and MS patients, respectively), are reported.

* significantly different from the group of sex and age matched healthy controls, $\mathrm{p}<0.0001$. 
In our group of 625 resting healthy controls we found a mean lactate value of $1.09 \pm 0.25 \mathrm{mmol} / \mathrm{l}$ serum (Panel A), with 615 of them having lactate ranging between 0.5 and $1.5 \mathrm{mmol} / \mathrm{l}$ serum and 10 outliers $(1.6 \%)$ with a lactate range of $1.76 \div 2 \mathrm{mmol} / \mathrm{l}$ serum. Independently on the clinical subtype and the EDSS score (Panel A), we detected a mean lactate value of $3.04 \pm 1.26 \mathrm{mmol} / \mathrm{l}$ serum in resting MS patients, i.e. about 2.8 times higher than that of controls $(\mathrm{p}<0.0001)$. As shown in the scatterplot of Panel B, 101 of the 613 MS patients (16.5\%) had serum lactate falling within the range of values determined in controls $(0.5 \div 2 \mathrm{mmol} / \mathrm{l}$ serum $)$. The remaining $502 \mathrm{MS}$ patients $(84.5 \%)$ had values higher than the maximal concentration of circulating lactate $(2.0 \mathrm{mmol} / \mathrm{l})$ recorded in one control healthy subject only. In MS patients, serum lactate ranged from $0.60 \mathrm{mmol} / \mathrm{l}$ to $7.56 \mathrm{mmol} / \mathrm{l}$. Analysis of the clinical and biochemical results considering MS patients as three separate cohorts (one for each recruiting center), are reported in Table 2. We found that the patients recruited at center 2 had a significantly lower EDSS scores compared to both center 1 and 3 (p < 0.05), with center 3 having the highest mean EDSS score. Additionally, center 3 recruited the highest $\%$ number of PP patients respect to both centers 1 and 2 . The very relevant finding is that the three centers had lactate values of $3.38 \pm 1.83 \mathrm{mmol} / \mathrm{l}$ serum (center 1$), 2.74 \pm 1.39 \mathrm{mmol} / \mathrm{l}$ serum (center 2) and $2.94 \pm 1.26 \mathrm{mmol} / \mathrm{l}$ serum (center 3), i.e. each of the three subset of MS patients had significantly higher lactate values than those found in healthy controls $(1.09 \pm 0.25$ $\mathrm{mmol} / \mathrm{l}$ serum; $\mathrm{p}<0.0001)$. Because of the differences in the EDSS score, lactate values recorded in patients recruited at center 2 were significantly lower than those measured in patients recruited in center 1 , thus reinforcing the concept of a correlation between serum lactate and clinical status in MS. 
Table 2. Clinical status and serum lactate in MS patients recruited in each per center.

\begin{tabular}{ccccccc}
\hline & $\begin{array}{c}\text { MS } \\
\text { (all) }\end{array}$ & RR & SP & PP & EDSS & $\begin{array}{c}\text { Serum } \\
\text { lactate }\end{array}$ \\
\hline Center 1 & $\mathrm{n}=281$ & $\begin{array}{c}202 \\
(71.9 \%)\end{array}$ & $\begin{array}{c}72 \\
(25.6 \%)\end{array}$ & $\begin{array}{c}7 \\
(2.5 \%)\end{array}$ & $3.82 \pm 2.23$ & $3 .[4] 31 \pm 1.83$ \\
\hline Center 2 & $\mathrm{n}=174$ & $\begin{array}{c}132 \\
(75.9 \%)\end{array}$ & $\begin{array}{c}37 \\
(21.2 \%)\end{array}$ & $\begin{array}{c}5 \\
(2.9 \%)\end{array}$ & $2.18 \pm 1.97$ & $2.81 \pm 1.39$ \\
\hline Center 3 & $\mathrm{n}=158$ & 96 & 44 & 18 & $4.21 \pm 1.79$ & $2.94 \pm 1.26$ \\
& & $(60.8 \%)$ & $(27.8 \%)$ & $(11.4 \% 9$ & & \\
\hline
\end{tabular}

MS = number of [5]all Multiple Sclerosis patients; PP = number of Primary Progressive patients (\% in each center), SP = number of Secondary Progressive patients (\% in each center); RR = number of Relapsing Remitting patients (\% in each center); EDSS = Extended Disability Status Scale score. In the EDSS and serum lactate columns, values represent mean \pm S.D and lactate is expressed in mmol/l serum.

To confirm these analytical results and to corroborate their relevance for the clinical biochemical monitoring of MS patients, we reported in Table 3 values of serum lactate determined in 150, randomly selected, samples of controls and 150, randomly selected, samples of MS patients, in which lactate was assayed either with the method described above or with the conventional methods rout[6]inely applied in the clinical biochemistry setting. Data indicate that no significant differences exist in the lactate values obtained with these two methods, thereby demonstrating the analytical validity of the present results. 
Table 3. Compariso[7]n of the analytical results obtained with a standard laboratory spectrophotometer and an apparatus in use in the clinical biochemistry setting and referring to serum lactate recorded in 150 randomly selected samples of controls and MS patients.

\begin{tabular}{|c|c|c|}
\hline & $\begin{array}{l}\text { Controls } \\
(n=150)\end{array}$ & $\begin{array}{c}\text { MS patients } \\
(n=150)\end{array}$ \\
\hline \multicolumn{3}{|l|}{ Serum lactate (mmol/l) } \\
\hline \multicolumn{3}{|l|}{ spectrophotometer } \\
\hline \multicolumn{3}{|l|}{ Serum lactate(mmol/l) } \\
\hline $\begin{array}{c}\text { Clinical biochemistry } \\
\text { apparatus }\end{array}$ & $1.11 \pm 0.25$ & $3.38 \pm 1.26^{\mathrm{a}}$ \\
\hline
\end{tabular}

Values are expressed as means \pm S.D.

${ }^{\mathrm{a}}$ significantly different from controls $(\mathrm{p}<0.0001)$.

To asses whether circulating lactate was correlated to clinical subtypes, MS patients were divided into RR, SP and PP and values of serum lactate in these groups were then calculated. As shown in Figure 2, all three subgroups of MS patients had higher values of lactate $(2.72 \pm 1.12,3.86 \pm 1.29$ and $3.32 \pm 1.16 \mathrm{mmol} / \mathrm{l}$ serum, respectively; $\mathrm{p}<0.0001$ ) than those detected in controls (Panel A). Significant differences were recorded in the comparisons between RR and SP ( $p<0.0001)$, RR 
versus PP ( $1<0.01)$, and SP versus PP $(\mathrm{p}<0.05)$. Importantly, the subgroup analysis with all progressive patients pooled together (SP and PP) demonstrated higher serum lactate levels $(3.77 \pm$ 1.28) compared to either RR-MS patients or control subjects ( $\mathrm{p}<0.0001$ for both comparisons), thus suggesting a correlation between MS subtype and serum values of this glycolytic end product (Figure 2, Panel B).
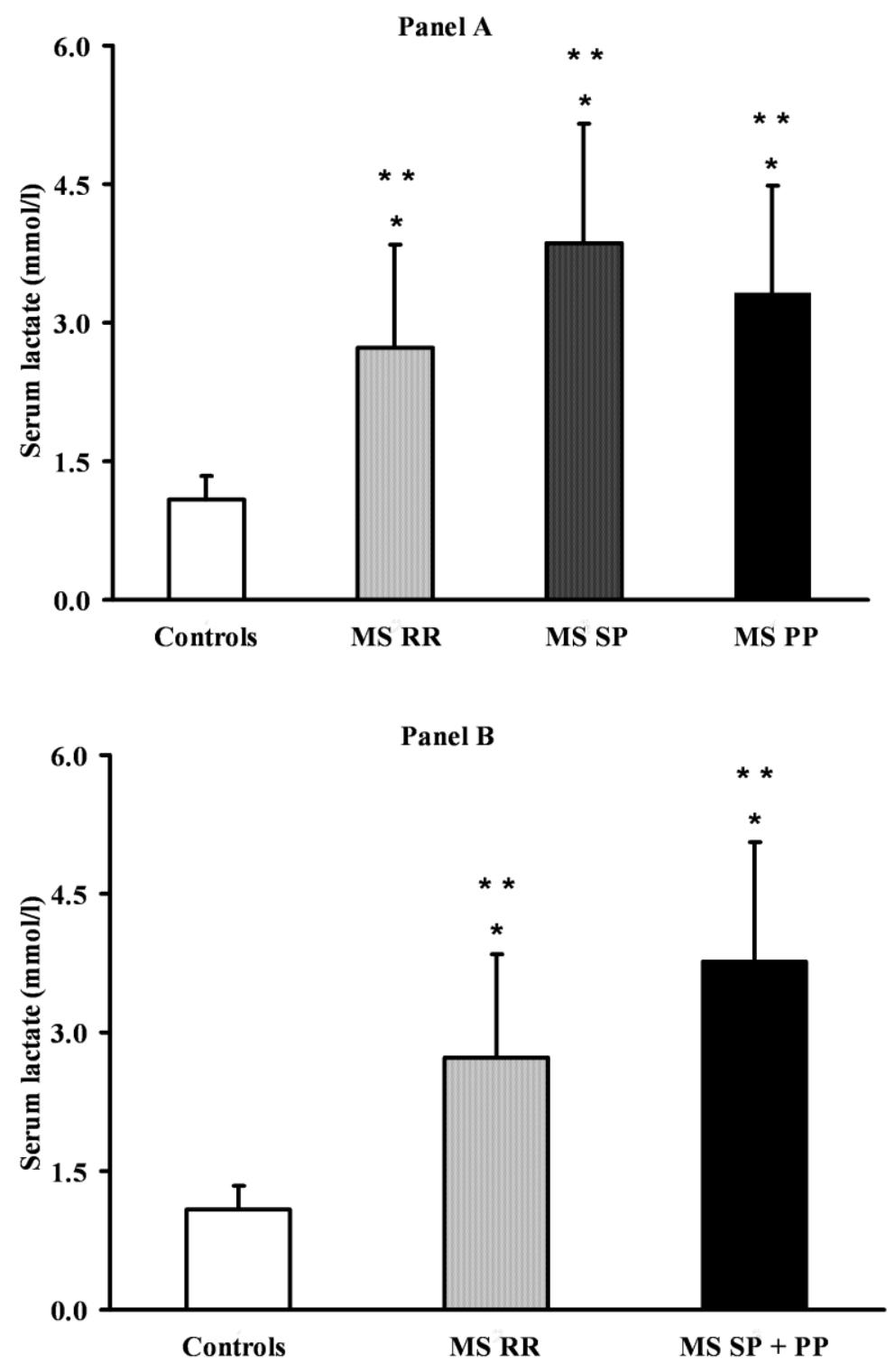
Figure 2. Concentration of serum lactate in controls and MS patients divided according to their different clinical subtype. In Panel A, patients were divided into three groups $(\mathrm{RR}=$ relapsing remitting; $\mathrm{SP}=$ secondary progressive; $\mathrm{PP}$ primary progressive) of very different size $(\mathrm{RR}=430 ; \mathrm{SP}=153 ; \mathrm{PP}=30)$ and possibly affecting statistical significance. In Panel $\mathrm{B}$, patients were divided into two groups $(\mathrm{RR}=430$ and $\mathrm{SP}+\mathrm{PP}=189)$. Values are reported as means, with standard deviations represented by vertical bars.

$*$ significantly different from the group of sex and age matched healthy controls, $p<0.001$.

** significantly different from the group of RR patients, $\mathrm{p}<0.0001$.

In order to investigate the correlation with the disease progression, values of serum lactate were plotted as a function of the EDSS scores (Figure 3).

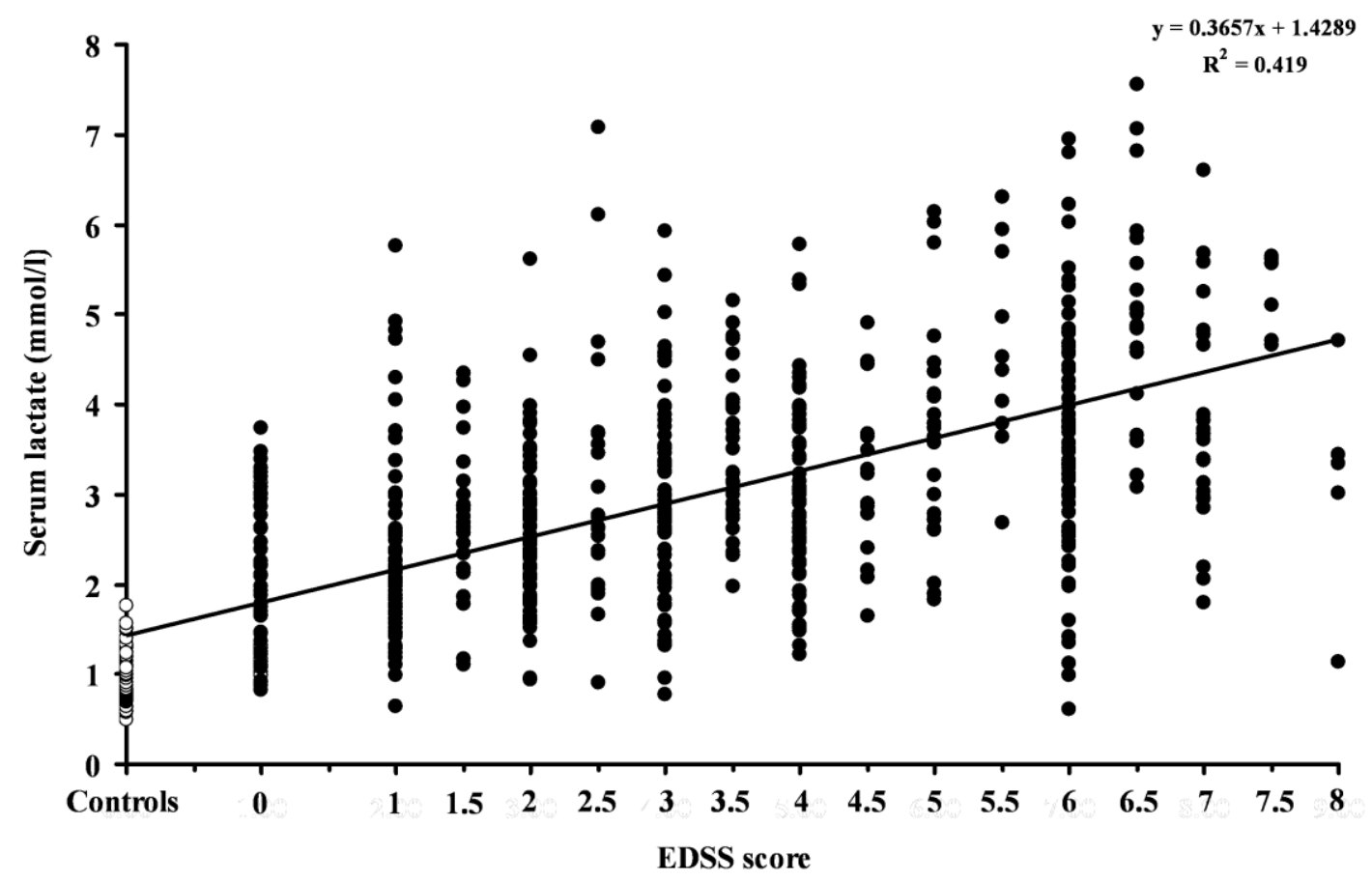

Figure 3. Dispersion graphic in which 76values of serum lactate of controls (randomly selected in the lactate values of the 625 healthy subjects) and of each of the 613 patients were plotted as a function of the EDSS scores. To the group of the 76 controls the real 0 on the $y$ axis was assigned. The value of the correlation coefficient $\left(R^{2}=0.419 ; p<0.0001\right)$ indicated that the two parameters were linearly correlated.

Since the number of subjects in the different EDSS subgroups did not exceed the 76 units, in order to avoid an excess weight of the control group in the calculation of the regression coefficients, we 
reduced the number of controls accordingly for the further statistical comparisons (analysis of regression and analysis of variance on the regression coefficients). Therefore, we randomly selected 76 values from the 625 lactate values of controls. This reduced in size control group (mean $=0.95 \pm$ 0.26 , median $=0.88$ ) had the same distribution (calculated using the Kolmogorov-Smirnov test) of the original larger control group and was used in the linear regression analysis. Lactate values in the control group were tabulated as the 0 values (no disease), whilst those in the MS patients were tabulated as the original EDSS score +1 . In Figure 3, the equation $(y=0.3657 x+1.4289 ; \mathrm{R}=$ 0.6473; $\left.\mathrm{R}^{2}=0.419 ; \mathrm{p}<0.0001\right)$ describing the best fitting regression line indicate a strong positive correlation linking serum lactate with disease progression (increase in EDSS scores), thus suggesting that the worsening of the clinical conditions of MS patients is accompanied by evident deteriorations in their cell energy metabolism. It is however worth underlining that a consistent $16.5 \%$ of MS patients had values of circulating lactate falling within the range of variability of control healthy subjects (Figure 1). These 101 "low lactate" MS patients were not found in MS patients scoring 3.5, 5.5, 6.5 and 7.5. on EDSS. Where present, they represented the 54.7\% (EDSS 0), 29.1\% (EDSS 1), 15.4\% (EDSS 1.5), 21.0\% (EDSS 2), 20.0 (EDSS 2.5), 16.1\% (EDSS 3), 17.2\% (EDSS 4), 10.0\% (EDSS 4.5), 6.0\% (EDSS 5), 11.1\% (EDSS 6), 9.7\% (EDSS 7) and 16.7\% (EDSS 8) of the respective EDSS class, with an evident clustering in the EDSS 0, characterized by the less severe clinical symptoms. It is important to note that without considering the 10 outliers of the control group, having lactate ranging between 1.76 and $2 \mathrm{mmol} / \mathrm{l}$ serum and representing the $1.6 \%$ of the 625 control values, the number of MS patients falling within the range of controls drops from 101 to $70(11.5 \%)$,

The box plot in Figure 4 shows the calculated best fitting linear regression on the medians of the different EDSS scores. According to the equation $\mathrm{y}=0.3935 \mathrm{x}+0.83\left(\mathrm{R}=0.9859 ; \mathrm{R}^{2}=0.972 ; \mathrm{p}<\right.$ 0.0001) it is possible to appreciate how medians of serum lactate in MS patients linearly increased as a function of the increase in EDSS score. It should be observed that, since in a box plot the 
abscissa is equally divided on the basis of the number of $\mathrm{x}$ values, patients with the intermediate EDSS scores $(1.5,2.5,3.5,4.5,5.5,6.5$ and 7.5$)$ were grouped with those of the upper EDSS class $(1.5-2,2.5-3,3.5-4,4.5-5,5.5-6,6.5-7$ and $7.5-8)$, in order to have equally spaced $x$ values. In addition, even in this case, the control group used in Figure $3(n=76)$ was considered as the 0 group (no disease) and the EDSS scores +1 were tabulated to perform the regression analysis.

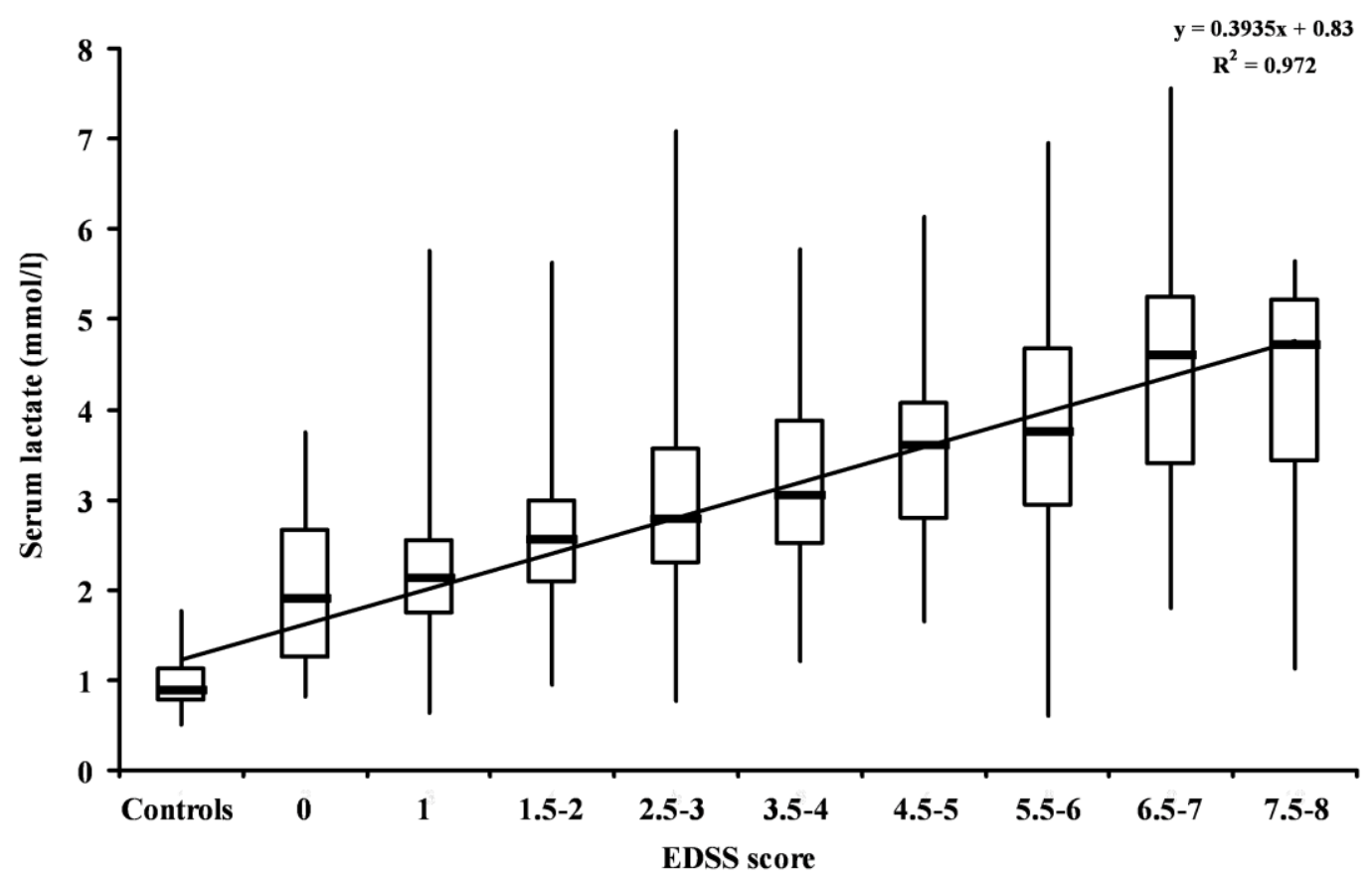

Figure 4. Box plot showing the linear correlation between the medians of the values of serum lactate recorded controls and the MS patients divided on EDSS scores. Values in controls refer to 76 serum lactate randomly selected in the values of the 625 healthy subjects. To plot this graph, patients with non integers EDSS scores were grouped with those of the nearest upper class, as indicated in the Figure. The value of the correlation coefficient $\left(R^{2}=0.9521 ; p<0.0001\right)$ indicated a statistically significant linear correlation. 


\section{DISCUSSION}

Data reported in the present study showed, for the first time to the best of our knowledge, that MS patients have remarkably elevated concentration of serum lactate respect to control healthy subjects. Furthermore, we also found that increased levels of circulating lactate in MS patients are strictly associated with the EDSS scores and with the clinical MS subtype (RR, SP, PP), thereby opening new perspectives for an ease, low cost, low invasive monitoring of the disease progression.

In the last decades, also thanks to the development of new imaging techniques such as ${ }^{1} \mathrm{H}-$ and ${ }^{31} \mathrm{P}$ MRS, several studies have clearly demonstrated significant metabolic changes in MS lesions and in normal appearing white matter suggesting that change in important metabolic functions may be implicated in the disease progression. In particular, decrease in NAA (Stromillo et al., 2013; Kirov et al., 2013; Steen et al., 2013), decrease in the total ${ }^{31} \mathrm{P}$ peak integrals and decrease in phosphodiesters/total ${ }^{31} \mathrm{P}$ (Husted et al., 1994), increase in total creatine (Kirov et al., 2013; Bagory et al., 2012), change in the activity of CPK-BB (Steen et al., 2010; Tur et al., 2012; Steen et al., 2013) are all suggestive of altered mitochondrial functions and neuronal energy state. In accordance to these findings, we found that MS patients show significant alterations of parameters indicative of impaired energy metabolism, such as uric acid, hypoxanthine, xanthine, creatinine, not only in CSF but also in serum/plasma (Amorini et al., 2009; Lazzarino et al., 2010; Tavazzi et al., 2011).

According to these strong indications, suggesting that MS is characterized by metabolic imbalance due to compromised mitochondrial functions, it is conceivable to expect that MS may cause increase in neuronal lactate production because of compensatory mechanisms. Data referring to possible changes in lactate in MS patients are controversial, since either increase (Simone et al., 1996; Lutz et al., 2007; Regenold et al., 2008) or decrease (Fonalledas Perelló et al., 2008) in CSF lactate have been reported. To increase the uncertainty about changes of this compound in relation to MS, it should be reminded that either elevated (Zaaraoui et al., 2010) or no change (Schocke et 
al., 2003) of brain lactate have been reported. In addition, data available in literature have mainly been obtained in relatively restricted cohorts of MS patients (Simone et al., 1996; Lutz et al., 2007; Regenold et al., 2008; Fonalledas Perelló et al., 2008). However, when lactate was found elevated in CSF of MS patients (Simone et al., 1996; Lutz et al., 2007; Regenold et al., 2008) no correlation with the disease progression on EDSS or with the clinical subtype (RR, SP, PP) has been demonstrated, thereby rendering doubtful the usefulness in the monitoring of this molecule in MS. Also, no systematic studies aimed at measuring lactate in serum/plasma of MS patients have been performed. Recently, it has been reported that levels of circulating lactate of patients with RR-MS were not different from those found in controls, even though results of this study have been obtained in a very small number of subjects $(\mathrm{n}=16$, for both controls and patients) even characterized by a low value of EDSS (Mähler et al., 2012).

Results reported in the present study, demonstrated about 3-times higher values of circulating lactate in MS patients than in control healthy subjects, let to hypothesize that either hyperglycolysis of sclerotic plaques or activated inflammatory cells might give raise to overproduction of lactate which only transiently remains into the CSF and rapidly diffuses into the blood stream. This sequence of events might not only explain the conflicting data of literature (Simone et al., 1996; Lutz et al., 2007; Regenold et al., 2008; Fonalledas Perelló et al., 2008; Zaaraoui et al., 2010; Schocke et al., 2003), but also why the increase in CSF lactate was not found to be correlated with EDSS or MS clinical subtype (Simone et al., 1996; Lutz et al., 2007; Regenold et al., 2008; Fonalledas Perelló et al., 2008; Zaaraoui et al., 2010; Schocke et al., 2003). It might also be possible that lactate in MS is not constantly overproduced but that it is subjected to fluctuations; this would render difficult to detect significant differences respect to controls when assaying lactate in the nervous tissue or in CSF. To clarify this issue, it should be important to perform longitudinal studies. It is conceivable that most of the exceeding lactate, intermittently or constantly, flows from CSF to the blood, thus explaining the significant differences recorded in the concentration of serum 
lactate, even when MS patients were divided into the RR, SP and PP clinical subtypes (Figure 2). In fact, we found that the SP and PP subgroups, characterized by higher mean EDSS scores (Table 1) due to higher neurodegenerative component leading to extensive neuroaxonal damage (Brück and Stadelmann, 2003), had higher circulating lactate than the RR- MS subgroup (Figure 2).

In our cohort of 613 MS patients, when plotting the concentrations of circulating lactate as a function of the EDSS scores a linear correlation has been obtained (Figures 3 and 4), notwithstanding the presence of some outliers particularly evident in the EDSS 2.5, 6 and 8 . Medians of $0.88 \mathrm{mmol} / \mathrm{l}$ serum for the control group and of 1.90 (EDSS 0), 2.12 (EDSS 1), 2.58 (EDSS 1.5-2), 2.78 (EDSS 2.5-3), 3.04 (EDSS 3.5-4), 3.60 (EDSS 4.5-5), 3.75 (EDSS 5.5-6), 4.61 (EDSS 6.5-7) and 4.71 (EDSS 7.5-8) for the MS patients divided on EDSS scores (Figure 4), strongly support the hypothesis of a metabolic impairment associated with the progression of the disease. However, it can be postulated that neither inflammation nor hyperglycolysis at the sclerotic plaques are sufficient to cause such a remarkable increase (up to 5 times the median value of controls) in the values of circulating lactate. According to previous data, MS patients showed an abnormal intramuscular component of fatigue (Sharma et al., 1995) and, those with mild disability, evidenced an increased cost of walking (Taylor et al., 2006; Franceschini et al., 2010). Both findings suggest the potential muscular involvement in MS, possibly caused by a metabolic imbalance of myocytes, and let to assume that part of the elevated circulating lactate detected in our cohort of MS patients is of muscular origin. It is certainly important to observe that not all MS patients had serum lactate higher than that found in controls. A consistent $16.5 \%$ of them had values of circulating lactate falling within the range of variability of control healthy subjects (Figure 1).

It is reasonable to suppose that the increase of lactate, may trigger (or may be the result of) a vicious circle in which the compensatory product of mitochondrial malfunctioning (lactate) may contribute to worsen mitochondrial activity itself. In fact, the intracellular acidification caused by increased 
lactate production may cause dramatic adverse effects on various and cell functions, including mitochondrial functions (Robergs et al., 2004).

One important additional observation that can be drawn from the results reported in this study is certainly related to the potential use of serum lactate determination to follow the disease progression in MS patients, as well as to monitor the eventual benefits of a pharmacological treatment. In spite of the very many efforts devoted to find new reliable biomarkers correlated with MS progression (Dujmovic 2011; Ziemann et al., 2011; Pravica et al., 2012; Singh et al., 2012; Tourdias and Dousset, 2013), to date clinicians are still waiting to know what should be assayed in order to have objective parameters, easily measurable, with which predicting the evolution of the disease or following the efficacy of drug administration. It may also be affirmed that clinicians are also waiting to know where this potential biomarker should be assayed: (post mortem) brain tissue, CSF, blood, extracellular fluid. At present, most of the proposed biomarkers are expensive in terms of cost of analysis (MRS, MRI, immunogenic profile, CD lymphocyte profile, antibodies detection, etc.), of invasiveness (lumbar puncture), of still uncertain reliability.

Detection of serum lactate is characterized by a very low cost, limited invasiveness, high automation, high reproducibility: the present results, indicating high correlation with the MS progression and MS clinical subtypes, suggest that lactate has all the characteristics of one of the best candidate to become one of the biomarkers of choice to follow MS progression and drug efficacy. It may be easily hypothesized that a frequent serum lactate determination would be of great help for physicians in knowing how an MS patient responds to a given therapy and how the disease is progressing. Also, improvement in the quality of results obtainable by the use of the so called "lactometer" for the self-made lactate measurement might allow an even more frequent measurement of this new potential biomarker. 


\section{ACKNOWLEDGEMENTS}

This work is dedicated to the memory of Professor Anna Paola Batocchi who had recently passed away. She dedicated her life to study multiple sclerosis and autoimmune diseases of the central nervous system. Without her initial input and idea, this study would have never been carried out.

\section{FUNDING}

This work has been funded in part by research funds of the Catholic University of Rome and the University of Catania. 


\section{REFERENCES}

Amorini AM, Petzold A, Tavazzi B, Eikelenboom J, Keir G, Belli A et al. Increase of uric acid and purine compounds in biological fluids of multiple sclerosis patients. Clin Biochem 2009; 42: 10016.

Andrews HE, Nichols PP, Bates D, Turnbull DM. Mitochondrial dysfunction plays a key role in progressive axonal loss in Multiple Sclerosis. Med Hypotheses 2005; 64: 669-77.

Artiss JD, Karcher RE, Cavanagh KT, Collins SL, Peterson VJ, Varma S et al. A liquid-stable reagent for lactic acid levels. Application to the Hitachi 911 and Beckman CX7. Am J Clin Pathol 2000; 114: 139-43.

Bagory M, Durand-Dubief F, Ibarrola D, Comte JC, Cotton F, Confavreux C et al. Implementation of an absolute brain 1H-MRS quantification method to assess different tissue alterations in multiple sclerosis. IEEE Trans Biomed Eng 2012; 59: 2687-94.

Brassat D. When does multiple sclerosis start? Three case reports and a review of the literature. Rev Neurol (Paris) 2012; 168: 846-51.

Brück W, Stadelmann C. Inflammation and degeneration in multiple sclerosis. Neurol Sci 2003; 24 Suppl 5: S265-7. 
Cambron M, D'Haeseleer M, Laureys G, Clinckers R, Debruyne J, De Keyser J. White-matter astrocytes, axonal energy metabolism, and axonal degeneration in multiple sclerosis. J Cereb Blood Flow Metab 2012; 32: 413-24.

Dujmovic I. Cerebrospinal fluid and blood biomarkers of neuroaxonal damage in multiple sclerosis. Mult Scler Int 2011; 2011: doi:10.1155/2011/767083.

Dutta R, McDonough J, Yin X, Peterson J, Chang A, Torres T et al. Mitochondrial dysfunction as a cause of axonal degeneration in multiple sclerosis patients. Ann Neurol. 2006; 59: 478-89.

Ehling P, Bittner S, Budde T, Wiendl H, Meuth SG. Ion channels in autoimmune neurodegeneration. FEBS Lett 2011; 585: 3836-42.

Fiorini A, Koudriavtseva T, Bucaj E, Coccia R, Foppoli C, Giorgi A et al. Involvement of oxidative stress in occurrence of relapses in multiple sclerosis: the spectrum of oxidatively modified serum proteins detected by proteomics and redox proteomics analysis. PLoS One 2013; 8: e65184.

Fonalledas Perelló MA, Politi JV, Dallo Lizarraga MA, Cardona RS. The cerebrospinal fluid lactate is decreased in early stages of multiple sclerosis. P R Health Sci J 2008; 27: 171-4.

Franceschini M, Rampello A, Bovolenta F, Aiello M, Tzani P, Chetta A. Cost of walking, exertional dyspnoea and fatigue in individuals with multiple sclerosis not requiring assistive devices. J Rehabil Med 2010; 42: 719-723. 
Haider L, Fischer MT, Frischer JM, Bauer J, Höftberger R, Botond G et al. Oxidative damage in multiple sclerosis lesions. Brain 2011; 134: 1914-24.

Hattingen E, Magerkurth J, Pilatus U, Hübers A, Wahl M, Ziemann U. Combined ${ }^{1} \mathrm{H}$ and ${ }^{31} \mathrm{P}$ spectroscopy provides new insights into the pathobiochemistry of brain damage in multiple sclerosis. NMR Biomed 2011; 24: 536-46.

Husted CA, Goodin DS, Hugg JW, Maudsley AA, Tsuruda JS, de Bie SH et al. Biochemical alterations in multiple sclerosis lesions and normal-appearing white matter detected by in vivo ${ }^{31} \mathrm{P}$ and ${ }^{1} \mathrm{H}$ spectroscopic imaging. Ann Neurol 1994; 36: 157-65.

Kirov II, Tal A, Babb JS, Herbert J, Gonen O. Serial proton MR spectroscopy of gray and white matter in relapsing-remitting MS. Neurology 2013; 80: 39-46.

Kurtzke JF. Rating neurologic impairment in multiple sclerosis: an expanded disability status scale (EDSS). Neurology 1983; 33: 1444-52.

Langemann H, Kabiersch A, Newcombe J. Measurement of low-molecular-weight antioxidants, uric acid, tyrosine and tryptophan in plaques and white matter from patients with multiple sclerosis. Eur Neurol 1992; 32: 248-52.

Lazzarino G, Amorini AM, Eikelenboom MJ, Killestein J, Belli A, Di Pietro V et al. Cerebrospinal fluid ATP metabolites in multiple sclerosis. Mult Scler 2010; 16: 549-54. 
Lublin FD, Reingold SC. Defining the clinical course of multiple sclerosis: results of an international survey National Multiple Sclerosis Society (USA) Advisory Committee on clinical trials of new agents in multiple sclerosis. Neurology 1996; 46: 907-11.

Lutz NW, Viola A, Malikova I, Confort-Gouny S, Audoin B, Ranjeva JP, et al. Inflammatory multiple-sclerosis plaques generate characteristic metabolic profiles in cerebrospinal fluid. PLoS One 2007; 2: e595.

Mähler A, Steiniger J, Bock M, Brandt AU, Haas V, Boschmann M, et al. Is metabolic flexibility altered in multiple sclerosis patients? PLoS One 2012; 7: e43675.

Miller E. Multiple sclerosis Adv Exp Med Biol. 2012; 724: 222-38.

Pandit A, Vadnal J, Houston S, Freeman E, McDonough J. Impaired regulation of electron transport chain subunit genes by nuclear respiratory factor 2 in multiple sclerosis. J Neurol Sci 2009; 279: 1420.

Polman CH, Reingold SC, Edan G, Filippi M, Hartung HP, Kappos L, et al. Diagnostic criteria for multiple sclerosis: 2005 revisions to the "McDonald Criteria". Ann Neurol 2005; 58: 840-6.

Pravica V, Popadic D, Savic E, Markovic M, Drulovic J, Mostarica-Stojkovic M. Single nucleotide polymorphisms in multiple sclerosis: disease susceptibility and treatment response biomarkers. Immunol Res 2012; 52: 42-52. 
Prineas JW, Parratt JD. Oligodendrocytes and the early multiple sclerosis lesion. Ann Neurol 2012; 72: $18-31$.

Regenold WT, Phatak P, Makley MJ, Stone RD, Kling MA. Cerebrospinal fluid evidence of increased extra-mitochondrial glucose metabolism implicates mitochondrial dysfunction in multiple sclerosis disease progression. J Neurol Sci 2008; 275: 106-12.

Robergs RA, Ghiasvand F, Parker D. Biochemistry of exercise-induced metabolic acidosis. Am J Physiol Regul Integr Comp Physiol 2004; 287: R502-16.

Schocke MF, Berger T, Felber SR, Wolf C, Deisenhammer F, Kremser C et al. Serial contrastenhanced magnetic resonance imaging and spectroscopic imaging of acute multiple sclerosis lesions under high-dose methylprednisolone therapy. Neuroimage 2003; 20: 1253-63.

Seven A, Aslan M, Incir S, Altıntaş A. Evaluation of oxidative and nitrosative stress in relapsing remitting multiple sclerosis: effect of corticosteroid therapy. Folia Neuropathol 2013; 51: 58-64.

Sharma KR, Braun J, Mynhier MA et al. Evidence of an abnormal intramuscular component of fatigue in multiple sclerosis. Muscle Nerve 1995; 18: 1403-1111.

Simone IL, Federico F, Trojano M, Tortorella C, Liguori M, Giannini P, et al. High resolution proton MR spectroscopy of cerebrospinal fluid in MS patients. Comparison with biochemical changes in demyelinating plaques. J Neurol Sci 1996; 144: 182-90. 
Singh V, Hintzen RQ, Luider TM, Stoop MP. Proteomics technologies for biomarker discovery in multiple sclerosis. J Neuroimmunol 2012; 248: 40-7.

Stadelmann C. Multiple sclerosis as a neurodegenerative disease: pathology, mechanisms and therapeutic implications. Curr Opin Neurol 2011; 24: 224-9.

Stangel M. Neurodegeneration and neuroprotection in multiple sclerosis. Curr Pharm Des 2012; 18 : 4471-4.

Steen C, D'haeseleer M, Hoogduin JM, Fierens Y, Cambron M, Mostert JP et al. Cerebral white matter blood flow and energy metabolism in multiple sclerosis. Mult Scler 2013; 19:1282-9.

Steen C, Wilczak N, Hoogduin JM, Koch M, De Keyser J. Reduced creatine kinase B activity in multiple sclerosis normal appearing white matter. PLoS One 2010; 5: e10811.

Stromillo ML, Giorgio A, Rossi F, Battaglini M, Hakiki B, Malentacchi G et al. Brain metabolic changes suggestive of axonal damage in radiologically isolated syndrome. Neurology 2013; 80: 2090-4.

$\mathrm{Su}$ K, Bourdette D, Forte M. Mitochondrial dysfunction and neurodegeneration in multiple sclerosis. Front Physiol. 2013; 4: 169: 1-10.

Su KG, Banker G, Bourdette D, Forte M. Axonal degeneration in multiple sclerosis: the mitochondrial hypothesis. Curr Neurol Neurosci Rep 2009; 9: 411-7. 
Tavazzi B, Batocchi AP, Amorini AM, Nociti V, D'Urso S, Longo S et al. Serum metabolic profile in multiple sclerosis patients. Mult Scler Int. 2011; 2011: 167156: 1-8.

Taylor NF, Dodd KJ, Prasad D, Denisenko S. Progressive resistance exercise for people with multiple sclerosis. Disabil Rehabil 2006; 28: 1119-1126.

Tourdias T, Dousset V. Neuroinflammatory imaging biomarkers: relevance to multiple sclerosis and its therapy. Neurotherapeutics 2013; 10: 111-23.

Trapp BD, Stys PK. Virtual hypoxia and chronic necrosis of demyelinated axons in multiple sclerosis. Lancet Neurol 2009; 8: 280-91.

Tur C, Wheeler-Kingshott CA, Altmann DR, Miller DH, Thompson AJ, Ciccarelli O. Spatial variability and changes of metabolite concentrations in the cortico-spinal tract in multiple sclerosis using coronal CSI. Hum Brain Mapp 2012; doi: 10.1002/hbm.22229: 1-12.

Zaaraoui W, Rico A, Audoin B, Reuter F, Malikova I, Soulier E et al. Unfolding the long-term pathophysiological processes following an acute inflammatory demyelinating lesion of multiple sclerosis. Magn Reson Imaging 2010; 28: 477-86.

Ziemann U, Wahl M, Hattingen E, Tumani H. Development of biomarkers for multiple sclerosis as a neurodegenerative disorder. Prog Neurobiol 2011; 95: 670-85. 